\title{
Recent studies of magnetic fields in Herbig Ae/Be stars ${ }^{\star}$
}

\author{
S. Hubrig ${ }^{1, \star \star}$, M. Schöller ${ }^{2}$, I. Ilyin ${ }^{1}$, B. Wolff ${ }^{2}$, C.R. Cowley ${ }^{3}$, M.A. Pogodin ${ }^{4}$, R.V. Yudin ${ }^{4}$, B. Stelzer ${ }^{5}$, \\ and Z. Mikulášek ${ }^{6}$ \\ ${ }^{1}$ Leibniz-Institut für Astrophysik Potsdam (AIP), An der Sternwarte 16, 14482 Potsdam, Germany \\ 2 European Southern Observatory, Karl-Schwarzschild-Str. 2, 85748 Garching, Germany \\ 3 Department of Astronomy, University of Michigan, Ann Arbor, MI 48109-1042, USA \\ 4 Pulkovo Observatory, Saint-Petersburg, 196140, Russia \\ 5 INAF-Osservatorio Astronomico di Palermo, Piazza del Parlamento 1, 90134 Palermo, Italy \\ 6 Department of Theoretical Physics and Astrophysics, Masaryk University, Brno, Czech Republic
}

Received 2011 Oct 3, accepted 2011 Oct 24

Published online 2011 Dec 12

\begin{abstract}
Key words stars: atmospheres - stars: individual (HD 97048, HD 101412, HD 150193, HD 176386, MWC 480) - stars: magnetic field - stars: pre-main sequence - stars: variables: general

New determinations of the mean longitudinal magnetic field for several Herbig Ae/Be stars are presented. The longitudinal magnetic field measurements of MWC 480 reveal the presence of a strong kG field, which was undetected in our previous low-resolution polarimetric observations with FORS 1 . The magnetic field geometries of Herbig Ae/Be stars studied with spectropolarimetric time series can likely be described by centred dipoles with polar magnetic field strengths of several hundred Gauss. A number of Herbig Ae/Be stars with detected magnetic fields were recently observed with X-shooter in the visible and near-IR as well as with the high-resolution near-IR spectrograph CRIRES. These observations are of great importance to understand the relation between the magnetic field topology and the physics of accretion flow and accretion disk gas emission.
\end{abstract}

(c) 2011 WILEY-VCH Verlag GmbH \& Co. KGaA, Weinheim

\section{Introduction}

Recent spectropolarimetric observations of several Herbig $\mathrm{Ae} / \mathrm{Be}$ stars indicate that magnetic fields are important ingredients of the intermediate-mass star formation process (e.g., Hubrig et al. 2004a, 2011a, 2006, 2007, 2009; Wade et al. 2007). Models of magnetically driven accretion and outflows successfully reproduce many observational properties of low-mass pre-main sequence stars, the classical T Tauri stars, but the picture is less clear for higher-mass stars. It is important to understand how the magnetic fields in Herbig $\mathrm{Ae} / \mathrm{Be}$ stars are generated and how these fields interact with the circumstellar environment, presenting a combination of disk, wind, accretion, and jets. Quite recently, magnetically controlled behaviour was found in spectroscopic and photometric variability of the strongly magnetic Herbig Ae star HD 101412 (Hubrig et al. 2011a, 2010). The few acquired UVES spectra of this star present variations in equivalent widths, radial velocities, line widths, line asymmetries, and mean magnetic field modulus over the rotation period of $42.1 \mathrm{~d}$. Obviously, magnetic field configurations are of utmost importance to understand the magnetospheres of Herbig Ae/Be stars. Since the accreting material has to be lifted

\footnotetext{
* Based on observations obtained at the European Southern Observatory, Paranal, Chile (ESO programmes 085.C-0137(A), 085.C-0137(A), 087.C-0124(A)) and SOFIN observations at the 2.56-m Nordic Optical Telescope on La Palma.

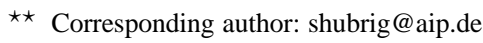

from the disk midplane to higher stellar latitudes near the stellar photosphere, coupling between the accreting plasma and a stellar magnetic field is required. Progress in understanding the disk-magnetosphere interaction can, however, only come from studying a sufficient number of targets in detail to look for patterns encompassing this type of premain sequence stars.

Series of mean longitudinal magnetic-field measurements were recently obtained at low resolution with the multi-mode instrument FORS 2 at the VLT for the Herbig Ae/Be stars HD 97048, HD 101412, HD 150193, and HD 176386. Magnetic fields of the order of 120-250 G were detected in these stars for the first time a few years ago during our visitor run with FORS 1 in May 2008 (Hubrig et al. 2009). The follow-up program for the search of magnetic field variations over the rotation cycle was allocated for observations with FORS 2 in 2010.

The previous spectropolarimetric observations of the star MWC 480 (= HD 31648) with FORS 1 revealed the presence of a weak longitudinal magnetic field $\left\langle B_{\mathrm{z}}\right\rangle=$ $87 \pm 22 \mathrm{G}$ and distinct circular polarisation signatures in spectral lines originating in the circumstellar (CS) environment (Hubrig et al. 2006, 2007). We report here on a few new high-resolution polarimetric spectra obtained with the SOFIN spectrograph installed at the Nordic Optical Telescope, which confirm the presence of a magnetic field in this star in both photospheric and CS lines. Further, we re- 
port on new X-shooter and CRIRES observations of a few Herbig Ae/Be stars.

\section{Magnetic field measurements and period determination}

Most of the presented magnetic field measurements are based on polarimetric spectra obtained with FORS 2 installed at the $8 \mathrm{~m}$ VLT telescope. Using a slit width of 0.4 , the achieved spectral resolving power of FORS 2 obtained with the GRISM 600B is about 2000. A detailed description of the assessment of the longitudinal magnetic-field measurements using FORS 2 is presented in our previous papers (e.g., Hubrig et al. 2004b, 2004c, and references therein). The mean longitudinal magnetic field, $\left\langle B_{\mathrm{z}}\right\rangle$, was derived using

$\frac{V}{I}=-\frac{g_{\mathrm{eff}} e \lambda^{2}}{4 \pi m_{\mathrm{e}} c^{2}} \frac{1}{I} \frac{\mathrm{d} I}{\mathrm{~d} \lambda}\left\langle B_{\mathrm{z}}\right\rangle$,

where $V$ is the Stokes parameter that measures the circular polarisation, $I$ is the intensity in the unpolarised spectrum, $g_{\text {eff }}$ is the effective Landé factor, $e$ is the electron charge, $\lambda$ is the wavelength, $m_{\mathrm{e}}$ the electron mass, $c$ the speed of light, $\mathrm{d} I / \mathrm{d} \lambda$ is the derivative of Stokes $I$, and $\left\langle B_{\mathrm{z}}\right\rangle$ is the mean longitudinal magnetic field. The longitudinal magnetic field was measured in two ways: using only the absorption hydrogen Balmer lines or using the entire spectrum including all available absorption lines.

Three spectropolarimetric observations of MWC 480 with $\mathrm{S} / \mathrm{N} \geq 200$ were obtained on 2009 December 30, 2010 December 14, and 2010 December 25 with the lowresolution camera $(R=\lambda / \Delta \lambda \approx 30000)$ of the échelle spectrograph SOFIN (Tuominen et al. 1999) mounted at the Cassegrain focus of the Nordic Optical Telescope (NOT). We used a $2 \mathrm{~K}$ Loral CCD detector to register 40 échelle orders partially covering the range from 3500 to $10000 \AA$ with a length of the spectral orders of about $140 \AA$ at $5500 \AA$.

A frequency analysis was performed on the longitudinal magnetic field measurements using a non-linear least-squares fit of the multiple harmonics utilizing the Levenberg-Marquardt method (Press et al. 1992) with an optional possibility of pre-whitening the trial harmonics. To detect the most probable period, we calculate the frequency spectrum and for each trial frequency we perform a statistical F-test of the null hypothesis for the absence of periodicity (Seber 1977). The resulting F-statistics can be thought of as the total sum including covariances of the ratio of harmonic amplitudes to their standard deviations, i.e. as a signal-to-noise ratio.

As we already reported in previous publications, the Herbig Ae star HD 101412 was found to be the first Herbig Ae star for which the rotational Doppler effect is small in comparison to the magnetic splitting and several spectral lines observed in unpolarised light at high dispersion are resolved into magnetically split components. The measured mean magnetic field modulus varies from 2.5 to $3.5 \mathrm{kG}$, while the mean quadratic field was found to vary in the range of 3.5 to $4.8 \mathrm{kG}$ (Hubrig et al. 2009, 2010). The rotation period of 42 days was determined from measurements of the longitudinal magnetic field and from photometry (Hubrig et al. 2011a). In spite of very clear evidence for the presence of a magnetic field in this star, the only previous measurement by other authors (Wade et al. 2007) showed contradicting results with $\left\langle B_{z}\right\rangle=512 \pm 111 \mathrm{G}$ using hydrogen lines and $\left\langle B_{z}\right\rangle=-527 \pm 313 \mathrm{G}$ using metal lines.

The derived ephemeris for the detected periods for the Herbig Ae/Be stars HD 97048, HD 150193, and HD 176386 are:

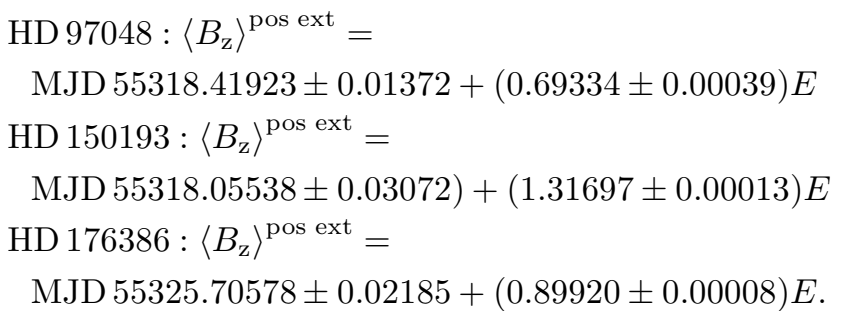

As an example, phase diagrams of the data for the Herbig Ae/Be stars HD 101412 and HD 150193 folded with the determined periods are presented in Fig. 1.

Assuming that the studied Herbig Ae/Be stars are oblique dipole rotators, the magnetic dipole axis tilt $\beta$ is constrained by $\left\langle B_{\mathrm{z}}\right\rangle^{\max } /\left\langle B_{\mathrm{z}}\right\rangle^{\min }=\cos (i+\beta) / \cos (i-\beta)$, where the inclination angle $i$ is derived from resolved observations of their disks. Our magnetic field model for the Herbig Ae star HD 101412 is described by a centered dipole with a polar magnetic field strength $B_{\mathrm{d}}$ between 1.5 and $2 \mathrm{kG}$ and an inclination of the magnetic axis to the rotation axis $\beta$ of $84 \pm 13^{\circ}$ (Hubrig et al. 2011a). For the star HD 97048, Lagage et al. (2006) used VISIR observations of mid-infrared emission of polycyclic hydrocarbons at the surface of the disk to determine $i=42.8 \pm 2.5^{\circ}$. Using this value, we obtain a magnetic obliquity of $\beta=84.8 \pm 5.0^{\circ}$ and the dipole strength $B_{\mathrm{d}}=720 \pm 120 \mathrm{G}$. The disk inclination of HD 150193, $i=38 \pm 9^{\circ}$, was determined by Fukagawa et al. (2003) using Subaru near-infrared imaging. With this value we find a magnetic obliquity $\beta=42 \pm 10^{\circ}$ and a dipole strength $B_{\mathrm{d}}=590 \pm 40 \mathrm{G}$.

Regarding HD 176386, the inclination angle $i$ is not known since both IR (Siebenmorgen et al. 2000) and farUV spectroscopy (Martin-Zaïdi et al. 2008) suggest that the circumstellar matter has the form of an envelope rather than the form of a disk. To constrain the magnetic field geometry of this star we determined $v \sin i=165 \pm 8 \mathrm{~km} \mathrm{~s}^{-1}$ from our NTT/EMMI spectrum obtained for this star in 1995 July 8 . The equatorial rotation velocity can be calculated using the relation $v_{\text {eq }}=50.6 R / P$, where $R$ is the stellar radius in solar units and $P$ is the rotation period in days. Taking into account the uncertainties in atmospheric parameter determinations for HD 176386, we adopt the radius $R=$ $3 \pm 0.5 \mathrm{R} \odot$. With this radius and the period $P=0.899 \mathrm{~d}$ we obtain $v_{\text {eq }}=169 \pm 28 \mathrm{~km} \mathrm{~s}^{-1}$, which leads to the poorly constrained $i=78 \pm 46^{\circ}$, magnetic obliquity $\beta=37 \pm 106^{\circ}$, 

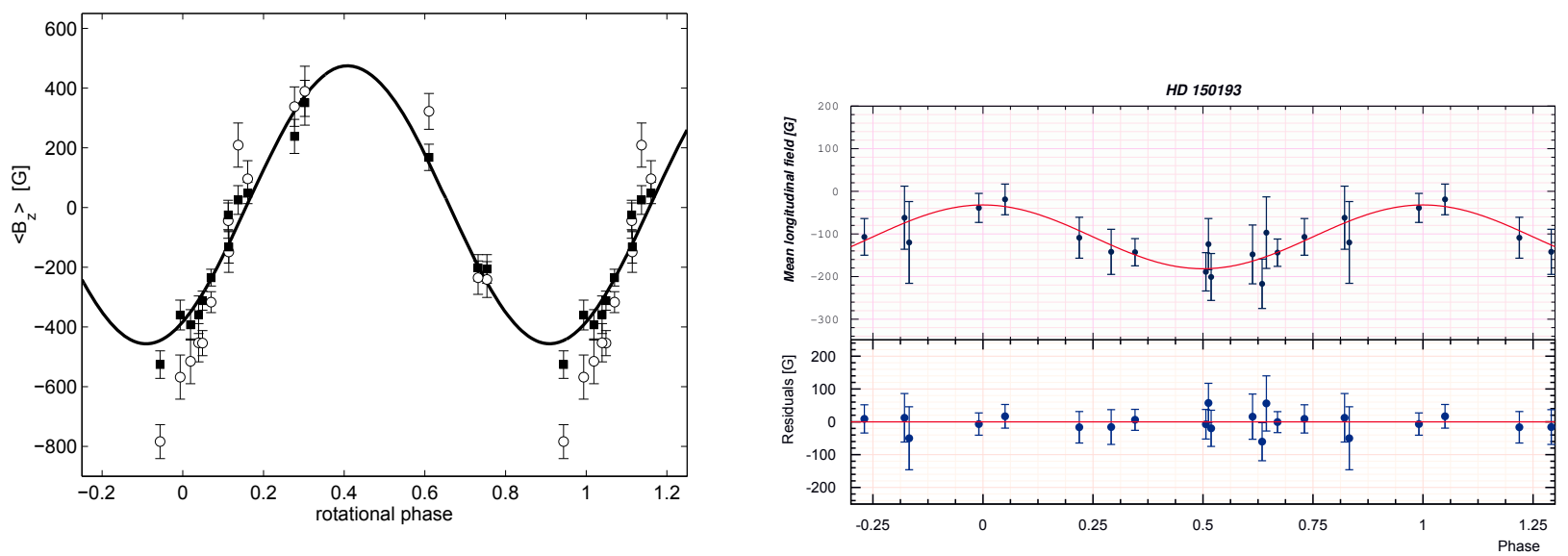

Fig. 1 (online colour at: www.an-journal.org) Left panel: phase diagram of HD 101412 with the best sinusoidal fit for the longitudinal magnetic field measurements using all lines (filled squares) and hydrogen lines (open circles). Right panel: phase diagram of HD 150193 with the best sinusoidal fit for the longitudinal magnetic field measurements using all lines. The residuals (Observed - Calculated) are shown in the lower panel.

and the dipole strength $B_{\mathrm{d}}=700 \pm 650 \mathrm{G}$ (Hubrig et al. 2011b).

The star MWC 480 exhibits a significant spectral variability, which hints at a strong interaction between the star and its CS environment. The temporal behaviour of the complex variable structure of $\mathrm{H} \alpha$, $\mathrm{He}$ I, and the $\mathrm{Na}$ I D was monitored in several previous studies (e.g., Beskrovnaya \& Pogodin 2004; Kozlova et al. 2007), which showed that the stellar wind is non-homogeneous and consists of several components, differing from each other by their velocities. Further, structural variations of the stellar wind have been found to correlate with changes in the accretion process in the envelope. In agreement with previous studies, our SOFIN spectra of MWC 480 show highly variable Na I $\mathrm{D}$ lines, but also the $\mathrm{Ca}$ II $\mathrm{H}$ and $\mathrm{K}$ lines display a complex structure, consisting of several components and pronounced variability. Magnetic field measurements of MWC 480 on SOFIN polarimetric spectra reveal a variable longitudinal magnetic field of the order of a few hundred Gauss up to one $\mathrm{kG}(-952 \pm 177 \mathrm{G})$. Weak magnetic fields of the order of several tens of Gauss were detected in the CS components of the $\mathrm{Na}$ I and $\mathrm{Ca}$ II doublet lines and can probably be considered as CS magnetic fields.

\section{X-shooter and CRIRES observations of magnetic Herbig Ae/Be stars}

All previously studied Herbig Ae/Be stars exhibit a singlewave variation in the longitudinal magnetic field during the stellar rotation cycle. These observations are usually considered as evidence of a dominant dipolar contribution to the magnetic field topology. Magnetospheric accretion theories traditionally consider simple $\sim \mathrm{kG}$ dipolar magnetic fields that truncate the disk and force in-falling gas to flow along the field lines. Similar to older studies of magnetospheric accretion on T Tauri stars, the recently obtained magnetic field models for Herbig Ae stars incorpo- rate dipole magnetic fields (e.g., Alecian et al. 2009; Hubrig et al. 2011a, 2011b). The assumption of the dominance of dipole fields is usually made for simplicity or due to the lack of available information about the true large-scale magnetic field topology of these stars. On the other hand, the recent work of Adams \& Gregory (2011) shows that high order field components may even play a dominant role in the physics of the gas inflow, as the accretion columns approach the star.

The rather new diagnostic He I $\lambda 1.083 \mu \mathrm{m}$ emission line is considered as probing inflow (accretion) and outflow (winds) in the star-disk interaction region of accreting $\mathrm{T}$ Tauri and Herbig Ae/Be stars. The uniqueness of this probe derives from the metastability of this transition and makes it a good indicator of wind and funnel flow geometry (Edwards et al. 2006). Further, according to Edwards et al., the He I line appears in emission for stronger mass accretion rates and in net absorption for low mass accretion rates. Modeling of this line allowed Gregory et al. (2011, in preparation) for the first time to study the influence of field topologies on the star-disk interaction. Their models use magnetic fields with an observed degree of complexity, as determined via field extrapolation from stellar magnetic maps.

In Fig. 2 we present our recent high-resolution CRIRES observations of the spectral regions containing the He I $\lambda 1.083 \mu \mathrm{m}$ line and the hydrogen recombination line $\mathrm{Pa} \gamma$ at $1.094 \mu \mathrm{m}$ over the rotation period of HD 101412. The rather strong variations of the line profile of the He I line indicate that the magnetic field of this star is likely more complex than a dipole field.

A variable behaviour of the He I $\lambda 1.083 \mu \mathrm{m}$ line was also discovered in our recent $\mathrm{X}$-shooter spectra of the magnetic Herbig Ae star HD 150193 (see Fig. 3).

Clearly, to properly understand how the magnetic field interacts with the inner accretion disk, the relation between the magnetic field topology and the physics of accretion 

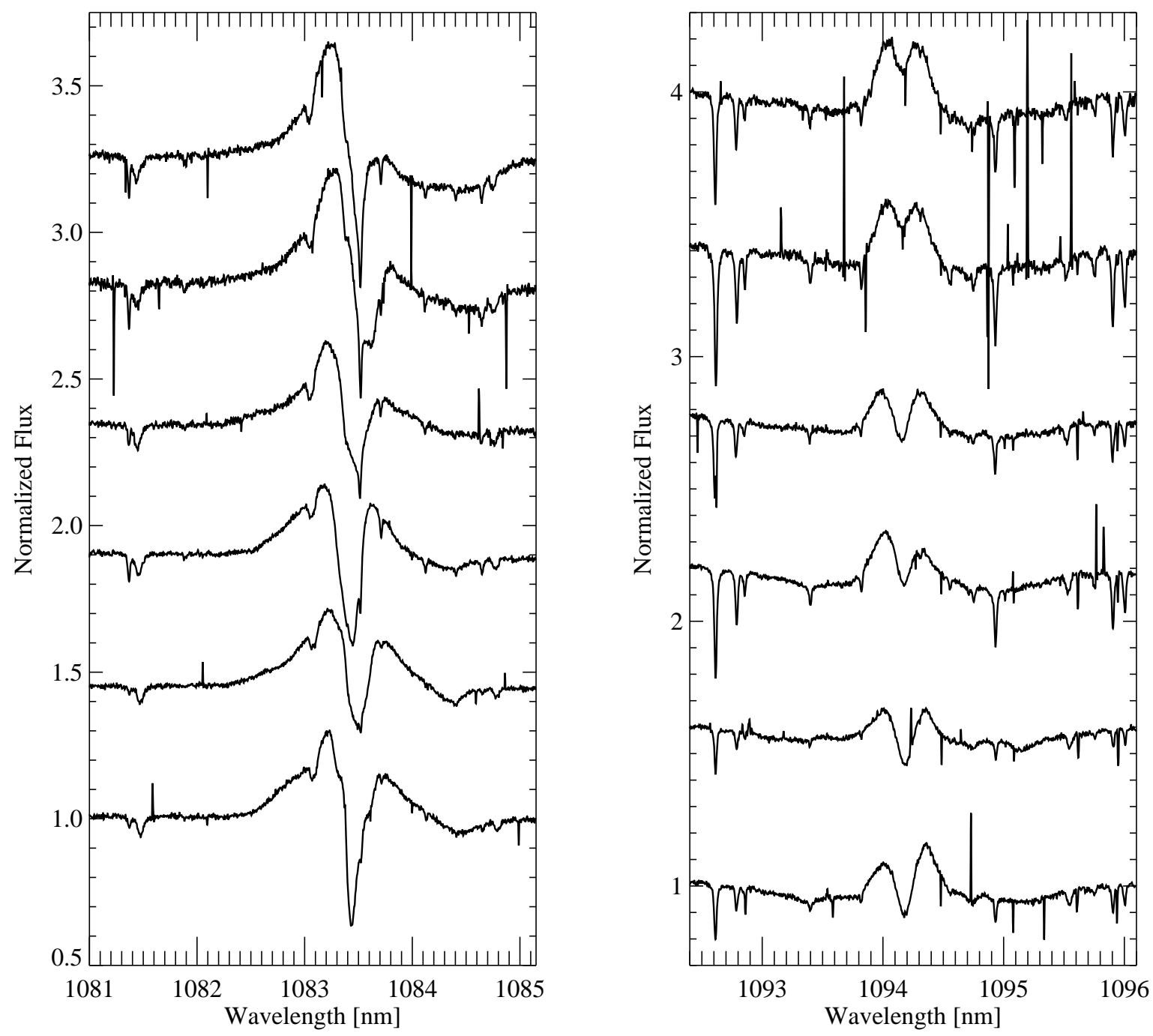

Fig. 2 Recent CRIRES observations of HD 101412. Left panel: the variability of the $\mathrm{He} \mathrm{I} \lambda 1.083 \mu \mathrm{m}$ line profile over the rotation period. Obviously, the field of HD 101412 appears more complex than just a dipole. Right panel: variations of the hydrogen recombination line $\mathrm{Pa} \gamma$ at $1.094 \mu \mathrm{m}$ at the same rotation phases. The $\mathrm{Pa} \gamma$ line at $1.094 \mu \mathrm{m}$ is frequently employed for calculating the mass accretion rate in the way presented by Gatti et al. (2008).

flow and accretion disk gas emission in Herbig Ae stars should be investigated in more detail using CRIRES or Xshooter near-IR spectral time series over the rotation periods. On the other hand, our experience shows that in most cases multi-wavelength photometry is quite useless to discover the rotation periodic variability of Herbig Ae stars. As an example, we show in Fig. 4 Hipparcos photometric data for V380 Ori, for which the rotation period $P=4.31276 \pm 0.00042 \mathrm{~d}$ was determined from magnetic field measurements (Alecian et al. 2009). As we show in this figure, no periodicity can be detected in the photometric data. We note that in the past only for three Herbig Ae stars, V380 Ori, HD 101412, and HD 135544B, rotation periods were known. They have been determined from direct measurements of longitudinal magnetic fields (for V380 Ori and HD 101412), photometry (for HD 101412), or radial ve- locities (for HD 135544B) (Alecian et al. 2009; Hubrig et al. 2011a; Müller et al. 2011).

\section{Discussion}

To understand the magnetospheres of Herbig Ae/Be stars and their interaction with the circumstellar environment presenting a combination of disk, wind, accretion, and jets, the knowledge of the magnetic field topology is indispensable. In our recent studies using low-resolution FORS 2 polarimetric spectra we showed that our magnetic field model for the Herbig Ae star HD 101412 is described by a centered dipole with a polar magnetic field strength $B_{\mathrm{d}}$ between 1.5 and $2 \mathrm{kG}$ and an inclination of the magnetic axis to the rotation axis $\beta$ of $84 \pm 13^{\circ}$ (Hubrig et al. 2011a). The fact that the dipole axis is located close to the stellar equatorial plane 


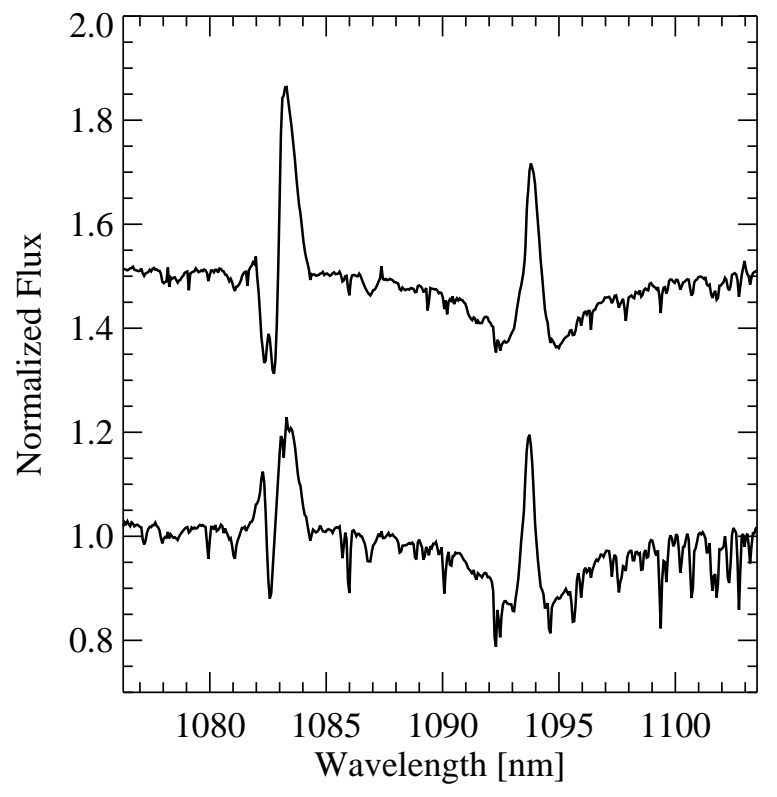

Fig. 3 Recent X-shooter observations of the He I $\lambda 1.083 \mu \mathrm{m}$ and $\operatorname{Pa} \gamma \lambda 1.094 \mu \mathrm{m}$ line profiles in the magnetic Herbig Ae star HD 150193 at two different rotation phases. The upper spectrum is shifted vertically for clarity.

is very intriguing in view of the generally assumed magnetospheric accretion scenario that magnetic fields channel the accretion flows towards the stellar surface along magnetic field lines. As was shown in the past (Romanova et al. 2003), the topology of the channeled accretion critically depends on the tilt angle between the rotation and the magnetic axis. For large inclination angles $\beta$, many polar field lines would thread the inner region of the disk, while the closed lines cross the path of the disk matter, causing strong magnetic braking, which could explain the observed unusually long rotation period of HD 101412. The best model for the magnetic field geometry of another Herbig Ae/Be star, V380 Ori, also indicates a rather large obliquity angle $\beta$ of $66 \pm 5^{\circ}$ (Alecian et al. 2009). Clearly, a representative sample of Herbig stars with carefully studied magnetic field geometry is urgently needed to properly understand the process of the magnetospheric accretion in these stars.

\section{References}

Adams, F.C., Gregory, S.G.: 2011, ApJ, in press, astro$\mathrm{ph} / 1109.5948$

Alecian, E., Wade, G.A., Catala, C., et al.: 2009, MNRAS 400, 354

Beskrovnaya, N.G., Pogodin, M.A.: 2004, A\&A 414, 955

Edwards, S., Fischer, W., Hillenbrand, L., Kwan, J.: 2006, ApJ 646,319

Fukagawa, M., Tamura, M., Itoh, Y., et al.: 2003, ApJ 590, L49

Gatti, T., Natta, A., Randich, S., et al.: 2008, A\&A 481, 423

Hubrig, S., Schöller, M., Yudin, R.V.: 2004a, A\&A 428, L1

Hubrig, S., Kurtz, D.W., Bagnulo, S., et al.: 2004b, A\&A 415, 661

Hubrig, S., Szeifert, T., Schöller, M., et al.: 2004c, A\&A 415, 685

Hubrig, S., Yudin, R.V., Schöller, M., Pogodin, M.A.: 2006, A\&A 446, 1089
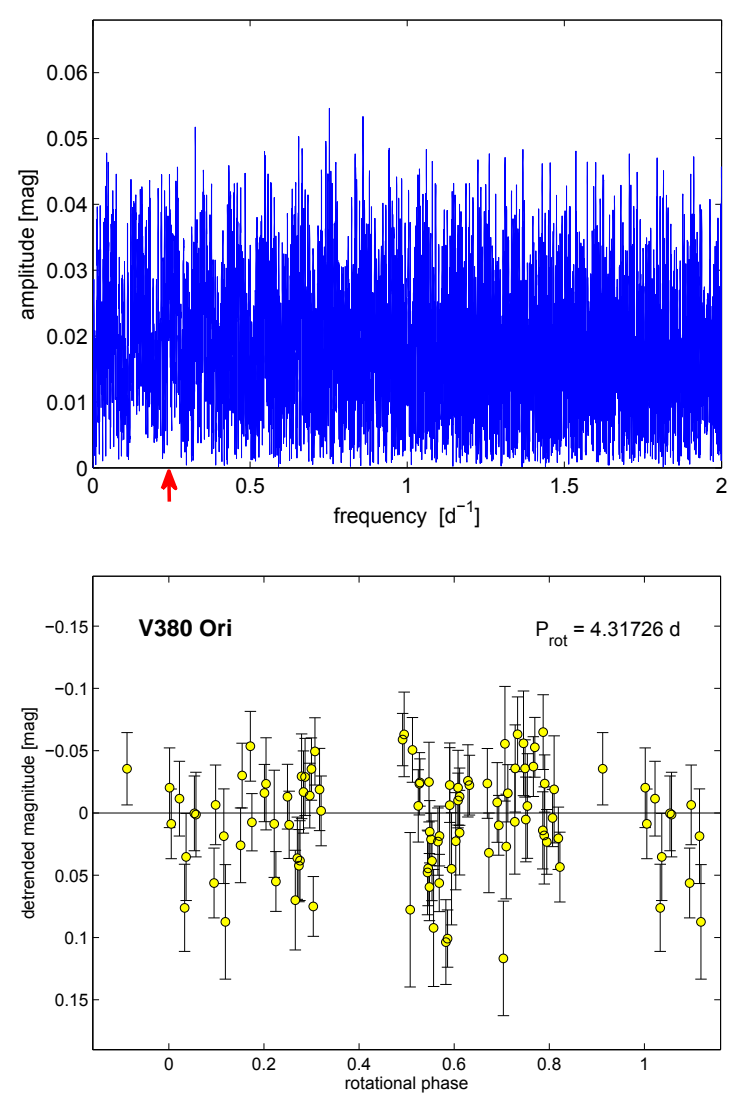

Fig. 4 (online colour at: www.an-journal.org) Frequency periodogram from Hipparcos observations of V380 Ori. The red arrow in this figure denotes the rotation period determined by Alecian et al. (2009). The lower panel presents the corresponding phase diagram. No periodicity is detected.

Hubrig, S., Pogodin, M.A., Yudin, R.V., et al.: 2007, A\&A 463, 1039

Hubrig, S., Stelzer, B., Schöller, M., et al.: 2009, A\&A 502, 283

Hubrig, S., Schöller, M., Savanov, I., et al.: 2010, AN 331, 361

Hubrig, S., Mikulášek, Z., González, J.F., et al.: 2011a, A\&A 525, L4

Hubrig, S., Schöller, M., Ilyin, I., et al.: 2011b, A\&A, in press

Kozlova, O.V., Alekseev, I.Yu., Shakhovskoi, D.N.: 2007, Astrophysics 50, 467

Lagage, P-O., Doucet, C., Pantin, E., et al.: 2006, Sci 314, 621

Müller, A., van den Ancker, M.E., Launhardt, R., et al.: 2011, A\&A 530, A85

Martin-Zaïdi, C., Deleuil, M., Le Bourlot, J., et al.: 2008, A\&A 484, 225

Press, W.H., Teukolsky, S.A., Vetterling, W.T., Flannery, B.P.: 1992, Numerical Recipes, 2nd ed., Cambridge University Press, Cambridge

Romanova, M.M., Ustyugova, G.V., Koldoba, A.V., et al.: 2003, ApJ 595, 1009

Tuominen, I., Ilyin, I., Petrov, P.: 1999, in: H. Karttunen, V. Piirola (eds.), Astrophysics with the NOT, p. 47

Seber, G.A.F.: 1977, Linear Regression Analysis, Wiley, New York

Siebenmorgen, R., Prusti, T., Natta, A., Müller, T.G.: 2000, A\&A 361,258

Wade, G.A., Bagnulo, S., Drouin, D., et al.: 2007, MNRAS 376, 1145 